\title{
Planetary nebulae with emission-line central stars
}

\author{
K. Gesicki ${ }^{1}$, A. A. Zijlstra ${ }^{2}$, A. Acker ${ }^{3}$, S. K. Górny ${ }^{4}$, K. Gozdziewski ${ }^{1}$, and J. R. Walsh ${ }^{5}$ \\ ${ }^{1}$ Centrum Astronomii UMK, ul. Gagarina 11, 87-100 Torun, Poland \\ e-mail: [Krzysztof.Gesicki;Krzysztof.Gozdziewski]@astri.uni.torun.pl \\ 2 School of Physics and Astronomy, University of Manchester, PO Box 88, Manchester M60 1QD, UK \\ e-mail: a.zijlstra@umist.ac.uk \\ 3 Observatoire astronomique de Strasbourg, 11 rue de l'Université, 67000 Strasbourg, France \\ e-mail: acker@astro.u-strasbg.fr \\ ${ }^{4}$ Copernicus Astronomical Center, ul.Rabianska 8, 87-100 Torun, Poland \\ e-mail: skg@ncac.torun.pl \\ 5 Space Telescope European Co-ordinating Facility, ESO, Karl-Schwarzschild-Strasse 2, 85748 Garching, Germany \\ e-mail: jwalsh@eso.org
}

Received 12 September 2005 / Accepted 29 December 2005

\begin{abstract}
The kinematic structure of a sample of planetary nebulae, consisting of 23 [WR] central stars, 21 weak emission line stars (wels), and 57 non-emission line central stars, is studied. The [WR] stars are shown to be surrounded by turbulent nebulae, a characteristic shared by some wels but almost completely absent from the non-emission line stars. The fraction of objects showing turbulence for non-emission-line stars, wels, and [WR] stars is $7 \%, 24 \%$, and $91 \%$, respectively. The [WR] stars show a distinct IRAS 12-micron excess, indicative of small dust grains, which is not found for wels. The [WR]-star nebulae are on average more centrally condensed than those of other stars. On the age-temperature diagram, the wels are located on tracks of both high and low stellar mass, while [WR] stars trace a narrow range of intermediate masses. Emission-line stars are not found on the cooling track. One group of wels may form a sequence wels-[WO] stars with increasing temperature. For the other groups, both the wels and the [WR] stars appear to represent several, independent evolutionary tracks. We find a discontinuity in the [WR] stellar temperature distribution and suggest different evolutionary sequences above and below the temperature gap. One group of cool [WR] stars has no counterpart among any other group of PNe and may represent binary evolution. A prime factor distinguishing wels and [WR] stars appears to be stellar luminosity. We find no evidence for an increase in the nebular expansion velocity with time.
\end{abstract}

Key words. planetary nebulae: general - stars: evolution

\section{Introduction}

Some central stars (cores) of planetary nebulae (PNe) show broad stellar-emission lines similar to Wolf-Rayet stars. Although superficially similar, they differ from classical WR stars in their degenerate structure, much lower masses, a wider range of temperatures, and limitation almost exclusively to carbon-rich stars (there are very few counterparts to the massive WN stars). Similar to their massive WC-star counterparts, they are deficient in hydrogen. They form a class named [WC]-type that historically was further divided into early [WCE] and late [WCL] groups. A less numerous class of PNe central stars show narrower and weaker emission lines than do the [WR]-type stars. These are named wels (weak emission-line stars), as defined in Tylenda et al. (1993).

The evolutionary status of the [WR]-type stars is still very uncertain, and it is unclear whether there is any evolutionary relation to the wels. Nebular data suggest an evolutionary sequence $[\mathrm{WC11}] \rightarrow[\mathrm{WO} 2]$ (Zijlstra et al. 1994; Acker et al. 1996; Peña et al. 2001), followed by PG 1159 stars (Werner et al. 1992). Acker \& Neiner (2003) propose a sequence: $[\mathrm{WC11}] \rightarrow[\mathrm{WC} 4] \rightarrow[\mathrm{WO} 4] \rightarrow[\mathrm{WO} 1]$. Parthasarathy et al. (1998) suggest that the wels are related to the PG 1159 stars: $[\mathrm{WCL}] \rightarrow[\mathrm{WCE}] \rightarrow$ wels $/ \mathrm{PG} 1159 \rightarrow \mathrm{PG} 1159$. But this sequence is by no means proved; the precise location of the wels in relation to the $[\mathrm{WR}]$ stars is under discussion (Marcolino \& de Araujo 2003). Peña et al. (2001) also argue against too closely identifying wels with PG 1159 stars (see also Koesterke 2001). Not all PG 1159 are hydrogen-poor (Dreizler et al. 1996), showing that not all PG 1159 stars will have evolved from [WR] stars. Neither are all wels hydrogen-poor: a dual evolutionary sequence may be expected.

The lack of hydrogen is often taken to indicate that the [WR] stars have undergone a late thermal pulse (or helium flash), either during the post-AGB evolution (a so-called late thermal pulse or LTP) or on the white dwarf cooling track (very late thermal pulse or VLTP: Herwig 2001; Hajduk et al. 2005). Following such a pulse, the star rejuvenates and retraces part of its earlier evolution. This predicts that nebulae around [WR] stars should be more evolved than around non-[WR] stars, but this is not confirmed by observations; the properties of the PNe around [WR] stars do not differ from those around other central stars (Górny 2001).

In this paper we discuss a much larger sample of [WR] stars and wels than has previously been available. We apply velocityfield analysis to locate the objects on derivatives of the HR diagram. In Sect. 2 we describe the methods applied for analysis of $\mathrm{PNe}$ with specific attention to automatic model fitting. Section 3 presents the 33 newly analyzed PNe. In Sect. 4 we discuss the 
nebular and stellar parameters for the full sample of $101 \mathrm{PNe}$. In Sect. 5 we discuss possible evolutionary relations.

Crowther et al. (1998) have refined the WC and the WO schemes used for WR stars and define a unified classification for massive WR and low-mass [WR] stars. Acker \& Neiner (2003) develope this scheme for a sample of 42 PN central stars, classified into [WO 1-4] and [WC 4-11] stars. This classification (used in the present paper) is based on the ionization level of the elements (depending on the wind-temperature of the PN core), essentially showing carbon lines for the coolest stars and oxygen lines for the hottest ones.

\section{Methods}

We derived information on the nebulae using a combination of line ratios, diameters, and high resolution spectra. The diameters and line ratios were used to fit a photo-ionization model. The model was constrained to reproduce the intensity distribution of images, if any were available. Line profiles were obtained from the high resolution spectra, and were fitted using the emissivity distributions of the photoionization model and assuming a velocity field. This was done using the Torun models (Gesicki et al. 1996, 2003). The velocity fields included separate contributions from expansion and turbulence. Turbulence always includes the instrumental broadening and the thermal broadening (calculated from the photoionization model), but some objects may require additional turbulence.

The models assume spherical symmetry. Some strongly bipolar objects cannot be fitted, because they show very irregular velocity profiles. But the majority of objects can be well reproduced with a spherical model. The model corrects for the size of the aperture used for the spectroscopy, and includes seeing effects.

The diameters and distances were adopted from the literature. The models find the density distribution, stellar temperature, and the velocity field. The density as function of radius is usually assumed in the shape of a reverted parabola; however, when images were available we compared the computed surface brightness and improved the run of the density. The stellar temperature assumes a black-body spectrum energy distribution - therefore we preferred to call it $T_{\mathrm{b}-\mathrm{b}}$ instead of $T_{\text {eff. }}$. This assumption can be challenged, especially for the [WR] central stars where the comparison between the stellar temperature $T_{*}$, obtained from non-LTE modelling, and $T_{2 / 3}$, which refers to the radius $R\left(\tau_{\text {Ross }}=2 / 3\right)$, shows differences. The differences are significant for hotter [WO] stars (Koesterke \& Hamann 1997), while smaller for cooler [WC] objects (Leuenhagen et al. 1996). The general tendency is always the same: the $T_{2 / 3}, T_{\text {eff }}$ or Zanstra temperatures are lower than $T_{*}$. Despite this discrepancy we apply the $T_{\mathrm{b}-\mathrm{b}}$ for further discussion since it has the advantage of providing a uniform measure of the temperature over a range of stellar classes.

The velocity distribution is assumed to vary arbitrarily and smoothly with radius. In Gesicki \& Zijlstra (2000) we compared the Torun model analysis to more traditional methods of deriving the expansion velocities from the spectra. For a long time it has been known that different observed lines result in different velocities (e.g. Weinberger 1989) indicating a velocity gradient. Our model has the advantage that it combines these data into a single velocity curve. It was not obvious earlier, but the Torun models have shown that even the shape of a single line can indicate a velocity gradient. Good velocity fields can be obtained if a larger number of lines are available. The resulting fields show detailed structure, with velocity peaks at the outer and sometimes at the inner radius of the nebula (Gesicki \& Zijlstra 2003). If fewer lines are available, the velocity field is less constrained, and simplifying assumptions need to be made. From the full velocity curve $V(r)$, we derive a single parameter characterizing the nebular expansion. We define the expansion velocity as a massweighted average over the nebula, $V_{\mathrm{av}}$. This parameter has been shown to be robust against the simplifying assumptions: it can be accurately determined even when the velocity field itself is uncertain (Gesicki et al. 2003). This allows us to define a kinematic age to the nebula.

\subsection{The genetic algorithm}

The Torun models were recently adopted to automate the search for a best-fit model, using an optimization routine. We applied the method widely known as the genetic algorithm. While it is still not a very popular optimization technique, it has proved to be very effective and robust in many problems, in particular for those of an astrophysical origin. For a review, we refer the reader to a paper by Charbonneau (1995), who is the author of the publicly available code PIKAIA for a genetic algorithm ${ }^{1}$ used for computations in this work.

The genetic algorithms were invented as an optimization technique mimicking the processes of biological evolution (e.g. Koza 1992). They lead to the selection and adaptation of life forms to the conditions of the natural environment. In this sense the evolution can be thought of as a powerful optimization algorithm. The genetic optimization starts with a set (population) of randomly chosen individuals, parameters of the mathematical model encoded in the "genomes". The whole parameter space of the problem creates the environment for these individuals. The likelihood of the survival of a given individual is determined by its "fitness" function $f$; usually, in the least-squares minimization, $f=1 / \sqrt{\chi^{2}}$. Then the fitness of a particular individual is a measure of the goodness of the fit to the studied data set. The population evolves through a number of generations. At each generation, the genetic algorithm evaluates $f$ resulting from each parameter set and processes information by applying genetic operators to the genomes like crossover, mutation, and selection. The best-fitted members of the population are used to produce a new generation, and the process continues until some convergence criteria is reached. Thanks to keeping the memory of the best-fitted individuals, the genetic algorithms are much more efficient than Monte Carlo-based search techniques. They are robust and especially well-suited for multi-dimensional problems, models possessing multiple local extrema, and discontinuities. They are non-gradient methods and thus especially well-suited for models that are dependent on their parameters in a sophisticated way. Details of the practical application of the technique are given in an excellent introduction by Charbonneau (2002).

The genetic optimization replaced the "trial and error" search for parameters applied in our earlier publications (e.g. Gesicki \& Zijlstra 2003; Gesicki et al. 2003). The algorithm appeared very effective in comparison with the earlier work. Because of random initialization of the population, the method is now free of initial biases. The search procedure again follows two steps. First PIKAIA searches for the values of the nebular mass and the stellar temperature and luminosity, all of which optimize the fit to the observables, before attempting to find the velocity field that

\footnotetext{
1 http://www .hao.ucar .edu/Public/models/pikaia/ pikaia.html
} 


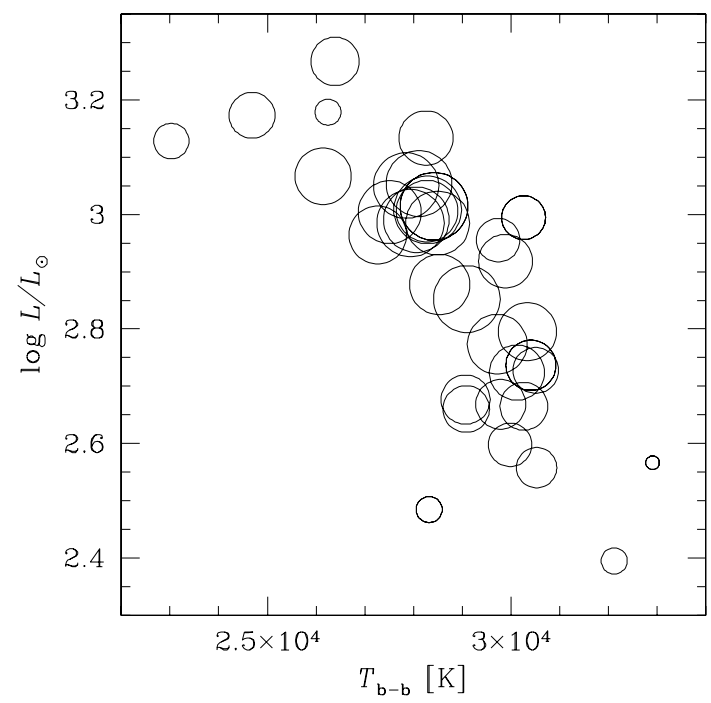

Fig. 1. The best fit luminosity and temperature for photoionization models for a single object (He 2-113, PNG 321.0+03.9) for 40 different PIKAIA runs. The larger the circle, the better the fit quality.

optimizes the fit to the line profiles. The following expression is optimized

$\chi^{2}=\frac{1}{N-p-1} \sum_{i=1}^{N}\left[\frac{O(i)-C(i)}{\sigma(i)}\right]^{2}$,

where $O(i)$ are $N$ observations $(i=1, \ldots, N), C(i)$ are model predictions dependent on $p$ parameters and $\sigma(i)$ are individual errors of the observations. In some cases considered in this work, $N$ is very small and the determination of uncertainties on the fitted parameters is difficult (and without much meaning in terms of the formal, statistical approach). Therefore we did not perform a formal error analysis of the results. Nevertheless, in a well-performed optimization search, the error analysis is as important as determining the fit parameters.

To check the possible degeneracy of the solutions and to obtain an idea about the errors, we collected the values to which the search process converged in many independent runs with the same start, and projected them onto chosen parameter planes. The distribution of these parameters gives insight into the significance of the minima, whether they can be well localized and fixed in the parameter space of the problem. In Fig. 1 we present an example of fitting the photoionization model for a single PN (He 2-113, PN G 321.0+03.9). Results are plotted for 40 different runs of PIKAIA, using the same set of observables. A scatter in the obtained parameters is seen: few of the points overlap. The size of the symbols corresponds to the fit quality. For this $\mathrm{PN}$ the temperature is better constrained than the stellar luminosity. The situation for other PNe is similar. For higher temperatures the models seem to be better constrained. We estimated the accuracy in $T_{\mathrm{b}-\mathrm{b}}$ as $\pm 2000 \mathrm{~K}$ and in $\log L / L_{\odot}$ as \pm 0.3 .

Similar tests were performed for the velocity procedure. When fitting a simple velocity field (linear or parabola-like), the PIKAIA routine repeats the results very well. More complicated velocity fields result in different shapes for different runs; however, the largest discrepancies appear in the least constrained parts of the velocity curves. Nevertheless the mass-averaged expansion velocity remains well-determined and is similar in all runs. From the distribution of models, we estimate the accuracy of $V_{\mathrm{av}}$ as about $\pm 2 \mathrm{~km} \mathrm{~s}^{-1}$.

\subsection{Observations}

A large number of PNe have been analyzed in previous papers using the Torun models. In most cases the velocity fields were determined from three lines (hydrogen $\mathrm{H} \alpha 6563 \AA$, [N II] $6583 \AA$, and [O III] $5007 \AA$ ) or fewer. To this sample we add 27 unpublished CAT observations, in the present paper covering only $\mathrm{H} \alpha$ and [N II] $6583 \AA$ and supplemented with recent echelle NTT observations of $6 \mathrm{PNe}$ with emission-line central stars. PNe with new data are listed in Table 1.

The ESO Coudé Auxiliary Telescope (CAT) was a subsidiary $1.4 \mathrm{~m}$ telescope feeding into the Coudé Echelle Spectrograph (CES) located at the neighbouring $3.6 \mathrm{~m}$ telescope. The CAT observations of 27 objects were performed during 1993 and 1994. The long camera was used, giving a spectral resolution of 60000 (corresponding to $5 \mathrm{~km} \mathrm{~s}^{-1}$ ). The slit width was $2^{\prime \prime}$. The observations used a long slit, sampled with $2^{\prime \prime}$ pixels. However, the spectra analyzed here used only the central row of pixels. (As the nebulae studied here are compact and the CAT was not an imaging-quality telescope, no spatial information was expected.) The spectrum covers one order of the echelle, covering $\mathrm{H} \alpha$ and the [N II] lines at $6548 \AA$ and $6853 \AA$, but no other nebular lines.

Six objects with emission-line central stars were observed with the ESO New Technology Telescope (NTT) during June 2001. The echelle spectra were obtained using ESO Multi Mode Instrument (EMMI), with grating 14 and cross disperser 3, giving a resolution of 60000 . The slit width was $1^{\prime \prime}$. The spectra covered the wavelength range 4300-8100 А. The spectra were summed over the slit length of $3^{\prime \prime}$. Exposure times were typically 120 s. Between 3 and 7 lines per object showed high enough S/N to be used in the velocity analysis. The details of the data reduction are given in Gesicki \& Zijlstra (2003).

The profile-fitting procedure requires specification of the centre of the emission line with zero velocity. We did not perform the full radial velocity correction. Instead we assumed the zero position in the centre of symmetry for the almost symmetric lines and usually located the zero position in the centre between the two maxima of the asymmetric lines. In the second case the presence of two (or more) spectral lines helped to locate the zero position, while the obviously hopeless cases were not analyzed.

\section{The modelled planetary nebulae}

Figures 2-4 present the observed and modelled nebular emission lines H I $6563 \AA$ and [N II] $6583 \AA$. Although for those indicated in the last column of the Table 1 six PNe more lines were used for model fitting ([O III] $5007 \AA$, and in some cases [O I] $6302 \AA$, [S II] $6732 \AA$, [S III] $6311 \AA$, He II $4686 \AA$ ), we present only the two lines for a consistent presentation. We do not draw the model radial distributions of the velocity, density, and surface brightness because our main results are the mass-averaged expansion velocities. The [WR]-subclass and wels classification was adopted from Acker \& Neiner (2003) and, for the lowest temperature [WC11] stars, from Górny et al. (2004). The summary of nebular and central star parameters are listed in Table 1. The last column indicates whether more than two lines were used for modelling. A few objects are discussed in the following.

PNG 002.6-03.4 (M 1-37), [WC11]“?”). The density structure is adopted to approximately agree with the image of Sahai (2000). The brightest nebular structure is the inner ellipsoidal ring $1.5 \times 2.5^{\prime \prime}$ that is modelled by our $2^{\prime \prime}$ sphere. The multipolar lobes, as well as the extended spherical halo, are beyond 
Table 1. The expansion velocities and other data concerning the nebulae and the central stars, based on observations described in this paper. The column "remarks" indicates how many emission lines were used for the analysis for PNe observed with EMMI.

\begin{tabular}{|c|c|c|c|c|c|c|c|c|c|c|c|c|}
\hline$\overline{\mathrm{PNG}}$ & Name & $\begin{array}{r}\log T_{\mathrm{b}-\mathrm{b}} \\
{[\mathrm{K}]}\end{array}$ & $\begin{array}{r}\log L \\
{\left[L_{\odot}\right]}\end{array}$ & $\begin{array}{l}\text { Dist. } \\
{[\mathrm{kpc}]}\end{array}$ & $\begin{array}{l}R_{\text {out }} \\
{[\mathrm{pc}]}\end{array}$ & $\begin{array}{l}M_{\text {ion }} \\
{\left[M_{\odot}\right]}\end{array}$ & $\begin{array}{l}V_{\mathrm{av}} \\
{[\mathrm{km} \mathrm{s}}\end{array}$ & $\begin{array}{l}\overline{V_{\text {trb }}} \\
-1]\end{array}$ & $\begin{array}{l}M_{\text {core }} \\
{\left[M_{\odot}\right]}\end{array}$ & $\begin{array}{r}t_{\mathrm{dyn}} \\
{[\mathrm{kyr}]}\end{array}$ & $\begin{array}{l}\text { Central } \\
\text { star }\end{array}$ & Remarks \\
\hline $000.9-04.8$ & M3-23 & 5.18 & 3.0 & 4.0 & .11 & .10 & 24 & 13 & 0.61 & 5.1 & & \\
\hline $002.6-03.4$ & M 1-37 & 4.40 & 3.9 & 8.0 & .04 & .08 & 27 & 0 & 0.60 & 1.7 & [WC11]? & \\
\hline $002.7-04.8$ & M 1-42 & 4.92 & 3.0 & 4.0 & .08 & .16 & 13 & 0 & 0.60 & 5.4 & & \\
\hline $003.6+03.1$ & M 2-14 & 4.64 & 3.0 & 8.0 & .05 & .06 & 17 & 10 & 0.60 & 2.9 & wels & 6 lines \\
\hline $004.6+06.0$ & H 1-24 & 4.56 & 3.8 & 7.0 & .08 & .13 & 24 & 0 & 0.58 & 3.7 & wels & \\
\hline $006.4+02.0$ & M 1-31 & 4.76 & 3.8 & 8.0 & .06 & .29 & 19 & 0 & 0.61 & 3.2 & wels & \\
\hline $008.2+06.8$ & He $2-260$ & 4.80 & 3.2 & 12.0 & .06 & .13 & 17 & 0 & 0.61 & 3.5 & & \\
\hline $211.2-03.5$ & M 1-6 & 4.60 & 3.5 & 4.0 & .03 & .05 & 24 & 0 & 0.62 & 1.4 & & \\
\hline $217.4+02.0$ & St 3-1 & 4.96 & 2.5 & 4.0 & .14 & .20 & 26 & 0 & 0.60 & 6.1 & & \\
\hline $232.0+05.7$ & SaSt 2-3 & 4.80 & 2.5 & 4.0 & .02 & .01 & 19 & 0 & 0.64 & 1.1 & & \\
\hline $232.4-01.8$ & M 1-13 & 5.04 & 2.9 & 4.0 & .10 & .19 & 14 & 0 & 0.60 & 6.5 & & \\
\hline $253.9+05.7$ & M 3-6 & 4.72 & 4.0 & 1.5 & .036 & .03 & 18 & 0 & 0.61 & 2.0 & wels & \\
\hline $258.1-00.3$ & He 2-9 & 4.73 & 2.9 & 1.5 & .015 & .01 & 25 & 0 & 0.65 & 0.7 & wels & \\
\hline $261.6+03.0$ & He $2-15$ & 5.17 & 3.0 & 2.2 & .13 & .32 & 22 & 0 & 0.71 & 6.4 & & \\
\hline $283.3+03.9$ & He 2-50 & 5.12 & 2.9 & 5.1 & .14 & .32 & 17 & 0 & 0.59 & 8.1 & & \\
\hline $292.4+04.1$ & PB 8 & 4.76 & 3.6 & 5.0 & .06 & .18 & 22 & 9 & 0.61 & 2.9 & [WC5-6] & \\
\hline $300.7-02.0$ & He 2-86 & 4.83 & 3.4 & 3.0 & .03 & .05 & 14 & 11 & 0.62 & 2.0 & [WC4] & \\
\hline $304.8+05.1$ & He 2-88 & 4.72 & 2.8 & 4.0 & .03 & .03 & 23 & 0 & 0.62 & 1.4 & & \\
\hline $309.0-04.2$ & He 2-99 & 4.49 & 2.7 & 4.0 & .16 & .25 & 45 & 10 & 0.57 & 4.6 & [WC9] & \\
\hline $316.1+08.4$ & He 2-108 & 4.51 & 3.8 & 4.0 & .10 & .14 & 21 & 9 & 0.57 & 5.0 & wels? & \\
\hline $321.0+03.9$ & He $2-113$ & 4.48 & 2.8 & 2.0 & .01 & .003 & 18 & 15 & 0.64 & 0.6 & [WC10] & \\
\hline $325.0+03.2$ & He 2-129 & 4.87 & 3.8 & 7.0 & .03 & .08 & 18 & 13 & 0.63 & 1.7 & & \\
\hline $325.8+04.5$ & He $2-128$ & 4.70 & 3.3 & 5.0 & .06 & .13 & 12 & 10 & 0.59 & 4.3 & & \\
\hline $347.4+05.8$ & H $1-2$ & 4.96 & 3.9 & 7.0 & .02 & .06 & 13 & 10 & 0.64 & 1.4 & wels & 3 lines \\
\hline $351.1+04.8$ & M 1-19 & 4.72 & 3.2 & 11.0 & .10 & .20 & 26 & 0 & 0.61 & 4.3 & wels & 5 lines \\
\hline $352.1+05.1$ & M 2-8 & 5.11 & 3.2 & 7.0 & .06 & .16 & 14 & 14 & 0.61 & 3.9 & [WO2-3] & 7 lines \\
\hline $355.9+03.6$ & Н 1-9 & 4.58 & 4.0 & 9.0 & .04 & .09 & 20 & 0 & 0.61 & 2.1 & & \\
\hline $356.1+02.7$ & Th 3-13 & 5.05 & 3.3 & 8.0 & .03 & .05 & 32 & 0 & 0.65 & 1.1 & wels & 3 lines \\
\hline $356.5-02.3$ & M 1-27 & 4.41 & 3.6 & 3.0 & .04 & .05 & 21 & 0 & 0.60 & 2.0 & [WC11]? & \\
\hline $357.1+03.6$ & M 3-7 & 4.72 & 3.2 & 4.0 & .05 & .07 & 23 & 0 & 0.61 & 2.4 & wels & 3 lines \\
\hline $357.4-03.2$ & M 2-16 & 4.95 & 3.3 & 7.0 & .09 & .25 & 21 & 14 & 0.61 & 4.5 & & \\
\hline $357.4-03.5$ & M 2-18 & 4.64 & 3.5 & 7.0 & .06 & .09 & 24 & 0 & 0.60 & 2.8 & & \\
\hline $359.1-02.3$ & M 3-16 & 4.57 & 3.8 & 7.0 & .08 & .14 & 30 & 0 & 0.59 & 3.1 & & \\
\hline
\end{tabular}

the scope of our analysis. We obtained a lower temperature than Zhang \& Kwok (1991) for this low-excitation nebula.

PNG 253.9+05.7 (M 3-6, wels). The radio image (Zijlstra et al. 1989) reveals a complicated structure with four maxima. Our spherical model is density bounded, but the line ratios are not well fitted and suggest a mixture of ionization and density boundaries. A small central cavity is indicated. The best velocity field for reproducing the multicomponent structure of the [N II] line shows multiple maxima. Considering the complicated nebular image, our model should be treated with caution, but because the spectral lines are almost symmetric, the mass-averaged expansion velocity is believed to be reliable.

PNG 321.0+03.9 (He 2-113, [WC 10]). The HST image (Sahai et al. 2000) reveals complex, highly aspherical structures, which makes spherical modelling doubtful. Nevertheless the average velocity still is a useful parameter. In our observations this $\mathrm{PN}$ is unresolved and the emission lines are symmetric.

\section{Derived parameters}

The newly analyzed data is combined with an earlier sample discussed in Gesicki et al. (2003). Together with the 33 new PNe, the full sample contains 101 objects; we removed three extragalactic PNe from 73 earlier objects, and two PNe were reanalyzed with the new spectra. The full sample contains 23 [WR], 21 wels, and 57 non-emission-line central stars.
Several objects were reclassified based on the work of Acker \& Neiner (2003) and Górny et al. (2004). The distinction between emission-line stars and non-emission-line stars depends on the depth of the available spectra. It is therefore possible that some of the non-emission-line stars would be classified as wels stars with deeper spectra. The effect of detectability of the stellar lines on the classification is discussed by Górny et al. (2004).

\subsection{Temperatures}

The subclass of the [WR] stars provides an indication of stellar temperature, but it cannot be used to predict unique temperatures. This can, for instance, be seen for the earlier subclasses (hotter stars) from the data in Acker \& Neiner (2003). De Araujo et al. (2002) show that deep spectra are needed for accurate association of a [WR] subclass. In this paper we use the photoionization (equivalent black-body) temperature $T_{\mathrm{b}-\mathrm{b}}$, rather than the [WR] subclass. This parameter has the further advantage of also being available for the non-[WR] stars. Figure 5 shows the relation between photoionization temperature and [WR] ([WO] and [WC]) subclass. There is a good relation overall, but the change in temperature is smaller for the later subclasses (8-11). One discrepant object is M2-43 (27.6 + 04.2) where the temperature is much higher than the subclass ([WC 7-8]) would suggest. The photo-ionization model fits the line ratios reasonably well, and the strong $[\mathrm{O}$ III] line with weak $[\mathrm{N}$ II] require a high temperature (Acker et al. 2002). Such high temperature was also obtained by 


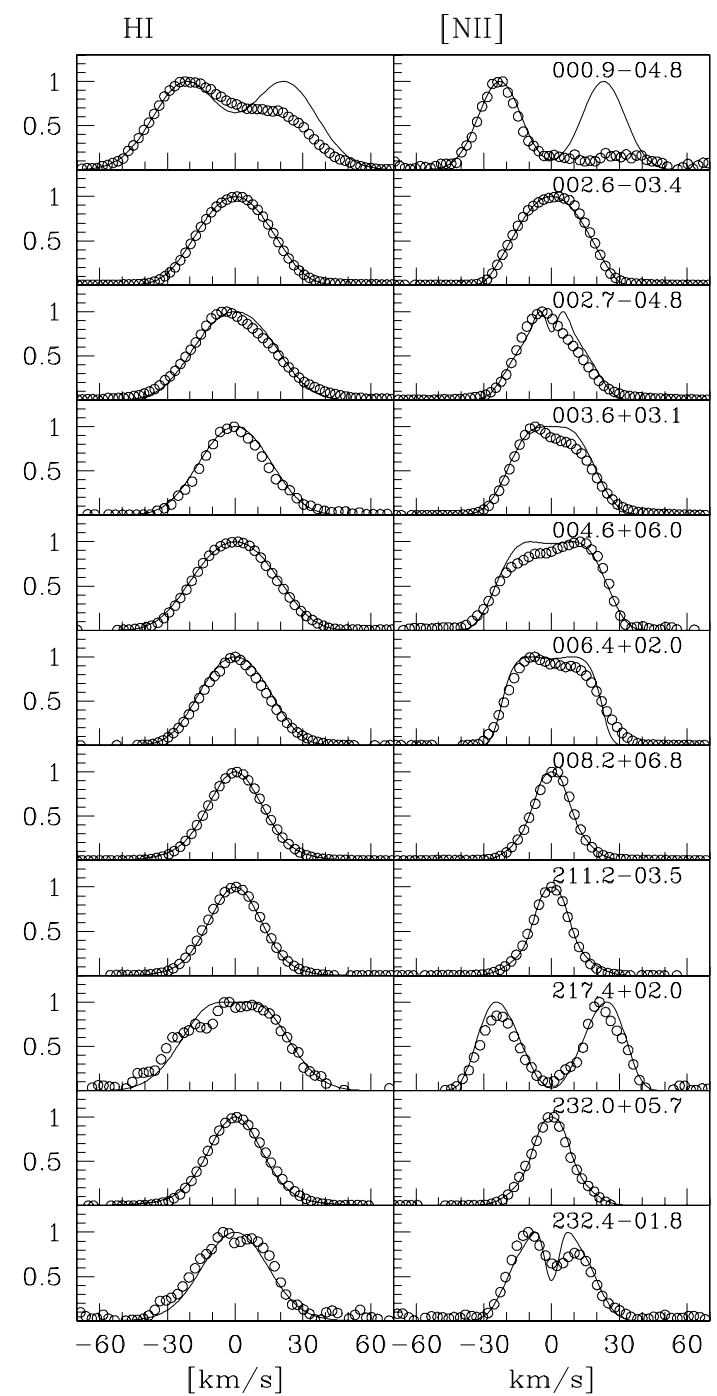

Fig. 2. The observed and modelled lines H I $6563 \AA$ and [N II] $6583 \AA$. The circles correspond to the observed profile, the line to the fitted model. The line fluxes are normalized to unity. The $X$-axis velocity scale given in the lowest boxes is the same for all plots.

non-LTE model analysis of Leuenhagen \& Hamann (1998). This object shows a dual dust composition (Górny et al. 2001). The [WC] subclass should be checked.

\subsection{Expansion velocities}

For the whole sample of $101 \mathrm{PNe}$, the average (and the median) value of the mass-averaged expansion velocities is $22 \mathrm{~km} \mathrm{~s}^{-1}$ with a standard error on the mean of $0.67 \mathrm{~km} \mathrm{~s}^{-1}$. The average $V_{\mathrm{av}}$ for [WR] PNe is $25 \pm 2 \mathrm{~km} \mathrm{~s}^{-1}$, while for wels it is $22 \pm 1 \mathrm{~km} \mathrm{~s}^{-1}$. The $1-\sigma$ difference in expansion velocity is much smaller than found in Peña et al. (2003a), who do find an average expansion velocity for non-WR PNe of $21 \mathrm{~km} \mathrm{~s}^{-1}$ (virtually the same as ours), but for [WR] PNe $36 \mathrm{~km} \mathrm{~s}^{-1}$. The difference can be explained by their method of estimating the velocity: they use the half-width at half maximum of the nebular lines and this is affected by the turbulence in the velocity fields (Acker et al. 2002).

It is natural to explain the higher-than-average expansion velocity of [WR] PNe in terms of stronger stellar wind acting on the swept-up shell. Mellema \& Lundqvist (2002) studied such

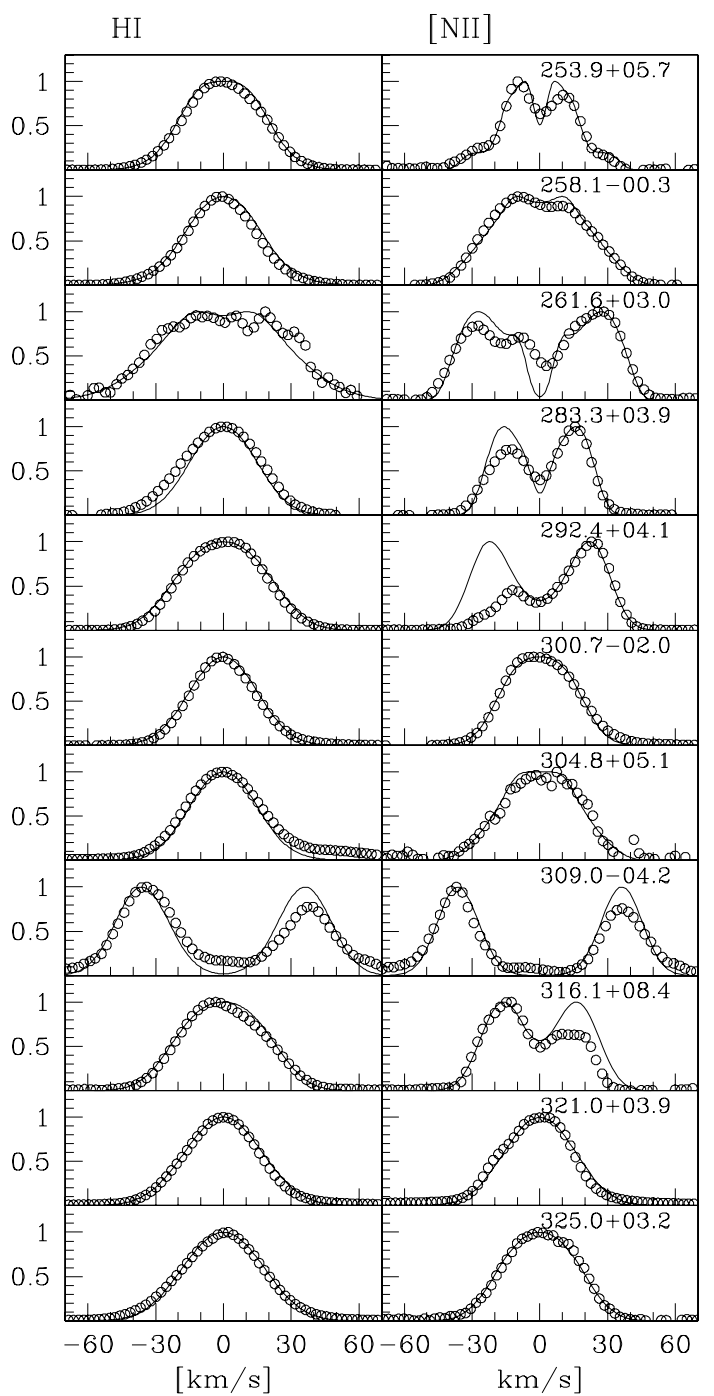

Fig. 3. The observed and modelled lines $\mathrm{H}$ I and [N II]. Continuation of Fig. 2.

interactions and confirm the above tendency providing an example of [WR] PN expansion of $21 \mathrm{~km} \mathrm{~s}^{-1}$ versus $17 \mathrm{~km} \mathrm{~s}^{-1}$ for non-[WR] PN. This difference is smaller than obtained by Peña et al. (2003a), but the values are rather comparable to ours.

Peña et al. (2003b) argue that the expansion velocities for evolved [WR] PNe are larger than for younger objects. In that paper the expansion velocity is defined as half-width at $1 / 10$ of the maximum intensity, a measure introduced by Dopita et al. (1988). At this intensity level, the emission lines are strongly broadened by a turbulent component common for [WR] PNe, so it is no surprise that our values (corrected for the turbulence) are lower. Peña et al. (2003b) arrive at their conclusion by plotting stellar temperatures versus expansion velocities. In our sample, no such effect is visible (Fig. 6, left panel), except that the three highest expansion velocities in the sample are from [WR] stars. The $V_{\text {exp }}$ and $T_{\mathrm{b}-\mathrm{b}}$ values are spread over the whole range. However, there is a trend for the [WR] stars to dominate towards the lower-right corner in the figure, i.e. lower values of $T_{\mathrm{b}-\mathrm{b}} / V_{\text {exp }}$. This trend looks very similar to the one from Fig. 3 of Peña et al. (2003b). However their [WR] PNe clustering in the upper-right corner is not all that similar.

Schönberner et al. (2005a) discuss their observations of $13 \mathrm{PNe}$ and conclude that expansion speed increases with the $T_{\mathrm{eff}}$ 


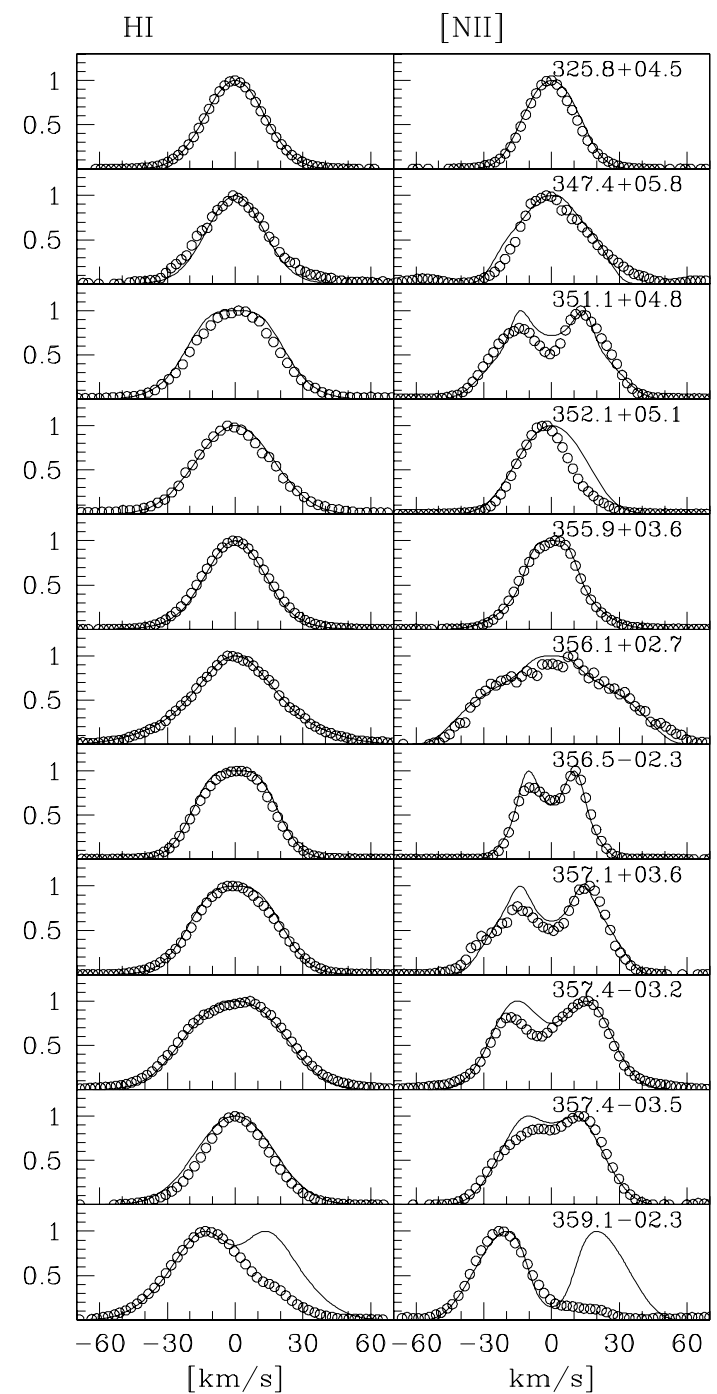

Fig. 4. The observed and modelled lines $\mathrm{H}$ I and [N II]. Continuation of Fig. 3.

of the central star, i.e. with the nebular age. Such a situation cannot be explained with simple models that assume an initial nebular density distribution described by a power law $\rho \propto r^{-\alpha}$ with a fixed index $\alpha$. Their conclusion is that the power-law index increases systematically with $T_{\text {eff }}$ (see their Fig. 12). We plot their model sequences for four values of the power law index in the left panel of Fig. 6. The indexes are from left to right: 2.00, 2.5, 3.0, and 3.25. Our data for $101 \mathrm{PNe}$ overlap with the lines (the velocities are not defined exactly in the same way). We do not confirm the Schönberner et al. (2005a) conclusion, but the range of power law indices that they derive provides a good fit to the spread seen in our sample. The trend in Fig. 6 for [WR] stars to dominate the distribution towards lower values of $T_{\mathrm{b}-\mathrm{b}} / V_{\exp }$, in combination with the slope of the model tracks, suggests that some [WR] PNe may have steeper initial density distributions than non-emission-line PNe.

\subsection{Dynamical ages}

The expansion velocity and radius of the nebula can be used to derive the age of the nebula. One can use the outermost radius and the maximum expansion velocity, but this is affected by the acceleration caused by the overpressure in the ionized region.

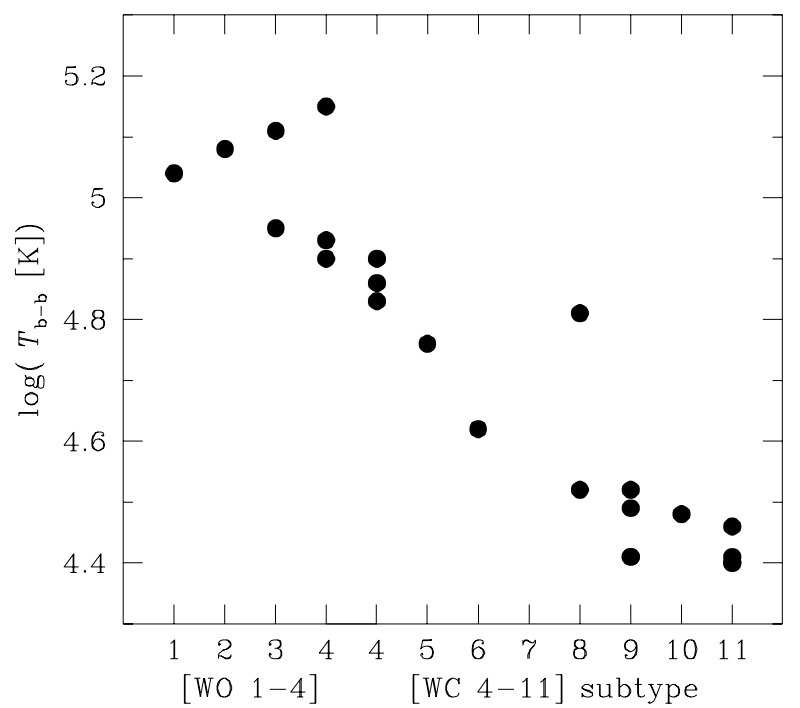

Fig. 5. Stellar photo-ionization temperatures versus [WO]-[WC] subclass.

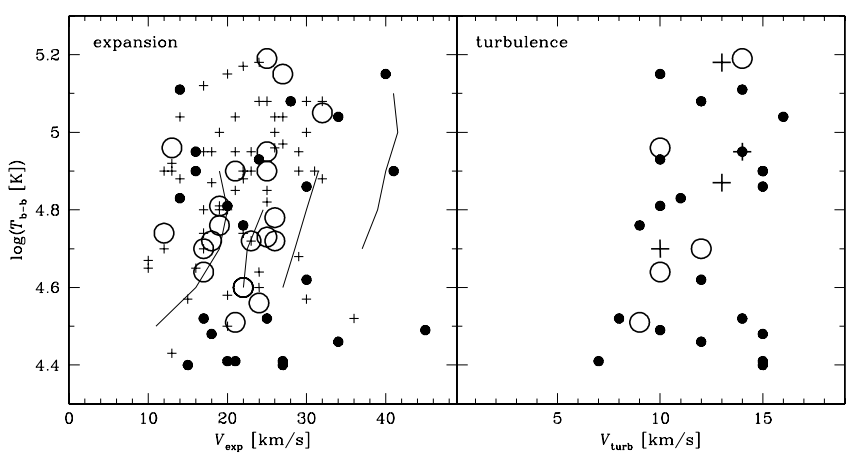

Fig. 6. The nebular expansion (left) and turbulent (right) velocities versus stellar temperature. The filled circles present [WR]-type PNe, open circles wels stars, and pluses show non-emission-line stars. In the left panel the lines represent the sequences modelled by Schönberner et al. (2005a), each line for a different initial density distribution. The accuracy of $V_{\mathrm{av}}$ is about $\pm 2 \mathrm{~km} \mathrm{~s}^{-1}$.

Instead we apply the mass-averaged velocity, and use for the radius 0.8 of the outer radius, roughly corresponding to the massaveraged radius. To account for the acceleration of the nebula we average between the current velocity and that of the original outflow velocities on the AGB. The procedure is described in Gesicki et al. (2003). We made a simplifying assumption about the AGB outflow velocity setting it equal to $10 \mathrm{~km} \mathrm{~s}^{-1}$ for all objects.

The dynamical ages can be directly compared between the different groups of stars. However, they may not be equal to the true ages of the nebulae, due to the various assumptions made. This problem was discussed, together with non-LTE analysis of some central stars of PNe. Rauch et al. (1994) found for the object K 1-27 that the dynamical age was more than one order of magnitude smaller than the evolutionary age. In another paper Rauch et al. (1999) analyzed four more PNe and obtained dynamical ages a few times larger than evolutionary ages. The nebular and stellar ages were compared on a much bigger sample by McCarthy et al. (1990). They found that the empirical ages were generally larger than the evolutionary ones, however their method for estimating dynamical ages was strongly criticized recently by Schönberner et al. (2005b). The contemporary situation is far from being conclusive. 
In this recent article we found nice support for our method for estimating nebular ages. Schönberner et al. (2005b) show that both radii and velocities increase gradually with time, which justifies our averaging between original and current velocity. For ionization-bounded models (dominant in our sample), the ages derived from ions [O III] and [N II] diverge in opposite directions from true ages, which supports our multi-line analysis where such effects compensate. For density-bounded PNe, the situation is even better since both lines result in the same age equaling the evolutionary one.

The dynamical age in combination with the stellar temperature gives the rate at which the central star increases in temperature. This rate is very sensitive to the core mass of the star, with more massive stars evolving much faster. To assign a core mass, we interpolate between published tracks (see e.g Górny et al. 1997; Frankowski 2003). For a given dynamical age and stellar temperature, we obtain the core mass (and luminosity). The dynamical ages and core masses are listed in Table 1. As for the ages, the mass determinations are internally consistent between different objects, but may show systematic offsets with respect to the true masses. As a check of the consistency of the procedure, the luminosities can be compared. In Gesicki et al. (2003), we found that the luminosities from photoionization modelling are in most cases smaller than those interpolated from evolutionary tracks. However this can be interpreted as leaking from the nebulae: the PN does not intercept and transform all stellar ionizing flux. This points to asymmetry or clumping.

\subsection{Turbulence}

For a number of nebulae, satisfactory fits to the velocity fields could not be obtained. In these cases we used enhanced turbulence, above what is expected from thermal broadening and instrumental resolution. Indicative of turbulence is that lines of all ions show Gaussian shapes of comparable width: thermal broadening gives much wider lines for the lightest element (hydrogen). Comparing the $\mathrm{H} \alpha$ with metal lines is crucial here because differences in thermal broadening between different metal ions are small. Therefore we searched for turbulent solutions only for those PNe with $\mathrm{H} \alpha$ and for at least one available metal line. Turbulence was indicated for 30 of the 101 nebulae in the full sample.

Gesicki \& Acker (1996) and Acker et al. (2002) find that [WR]-type PNe are characterized by strong turbulent motions while non-emission-line PNe are not. This general tendency is confirmed in the present analysis, but there are some exceptions. Among the wels we find both turbulent and non-turbulent objects. Our full sample contains 21 wels of which 5 show turbulence and 16 do not. Of these 21 objects, 10 come from the new observations described here (3 turbulent and 7 non-turbulent).

We also find well-determined, turbulent solutions for $4 \mathrm{PNe}$ neither classified as [WR] nor wels (M 2-16, M 3-23, He 2-128, He 2-129). Not all [WR]-type PNe in the new sample show turbulent motions: M 1-27 and M 1-37 have good solutions, without requiring turbulence. The two non-turbulent [WR] PNe are both classified by Górny et al. (2004) as “[WC11]?". They are the coolest objects in our sample. However, the strong correlation between emission-line stars and turbulence remains: the fraction showing turbulence for non-emission-line stars, wels, and [WR] stars is $7 \%, 24 \%$, and $91 \%$, respectively.

Uncertainties in the [WR] identifications may affect these fractions. The turbulent non-WR PNe could have emission-line central stars. This is unlikely for M2-16, a well-studied, highly metal-rich PN (Cuisinier et al. 2000). He 2-128 and He 2-129 were studied spectroscopically by Dopita \& Hua (1997): the non-WR identification cannot be attributed to a lack of observations, but faint stellar lines may have been missed. Some objects may have too few nebular lines to be able to detect turbulence: a velocity gradient broadens a single line profile similar to turbulence - we only accepted turbulent solutions where they clearly fitted the line shapes better than non-turbulent ones. Deeper spectra would help here.

The onset of the turbulence and the [WR] phenomenon may not be completely simultaneous. However, we found no evidence that the turbulence increases for more evolved objects: Fig. 6 (right panel) shows no relation between turbulence and stellar temperature.

In trying to understand why the presence of turbulence in wels $\mathrm{PNe}$ is so mixed, let us consider a possibility that a PN can switch between turbulent and non-turbulent regimes during its lifetime. The transition time for this should be comparable to the shock travel time across the nebula. Assuming a radius of $0.1 \mathrm{pc}$ and isothermal sound speed of $10 \mathrm{~km} \mathrm{~s}^{-1}$ we obtain a time of $10^{4}$ years. Perinotto et al. (2004) state that the shock front velocity can be 3-4 times faster than the isothermal sound speed: this reduces the time to a few thousand years. This is comparable to the ages of our PNe. In our sample we have a couple of (compact) objects with an age of about 1000 years that have already developed turbulence. Acker et al. (2002) propose that turbulence in PNe is triggered or enhanced by [WR] stellar-wind inhomogeneities. The nebulae surrounding very active [WR] cores are turbulent. If the less active wels with much weaker winds could change their wind characteristics, they would develop turbulence, or exhibit decaying turbulence, over the lifetime of the PN. Thus, the mixed occurrence of turbulence in wels is compatible with a transitory status, not an unlikely [WR]-star progenitor, provided the life time of the phase does not exceed $\sim 10^{3} \mathrm{yr}$.

\subsection{IRAS colours}

Planetary nebulae are strong infrared emitters, due to their heated dust. The dust colours follow the evolution: as the nebula expands, the dust cools and the dust colours redden. Zijlstra (2001) has shown that [WR] stars are stronger infrared emitters than other PNe, with a tendency for bluer colours. The brightest infrared PNe are the IR-[WR] stars, a subgroup of the cooler [WC] stars.

Figure 7 shows the colour-colour diagram for the present sample. Different symbols distinguish the [WR] stars (filled circles), the wels (open circles), and the non-emission-line stars (pluses). In addition, overplotted star symbols indicate turbulent nebulae. We excluded the confused source M2-6 where the IRAS colours appear to be those of a nearby AGB star. The boxes are source-classification regions of Zijlstra et al. (2001). Planetary nebulae fall in box II. The cooler [WC] stars are found mainly towards the left in the diagram, in three cases with colours like those of (young) post-AGB stars. (The three objects are BD +30 3639, M 4-18, and He 2-142.) The wels are shifted to redder colours, and their distribution is similar to those of normal $\mathrm{PNe}$. Note that the cooler non-emission-line stars in our sample have 60/25-micron colours similar to the cool [WC] PNe, but their 25/12-micron colours are very different.

We constructed dust models following the nebular expansion occurring as the star heats up. We used an $r^{-2}$ wind, with the model and recipe of Siebenmorgen et al. (1994). The model calculates the IRAS fluxes at any particular time. The results are shown in Fig. 7 for carbon-rich (long dashes) and oxygenrich (short dashes) nebular dust. The evolutionary progression 


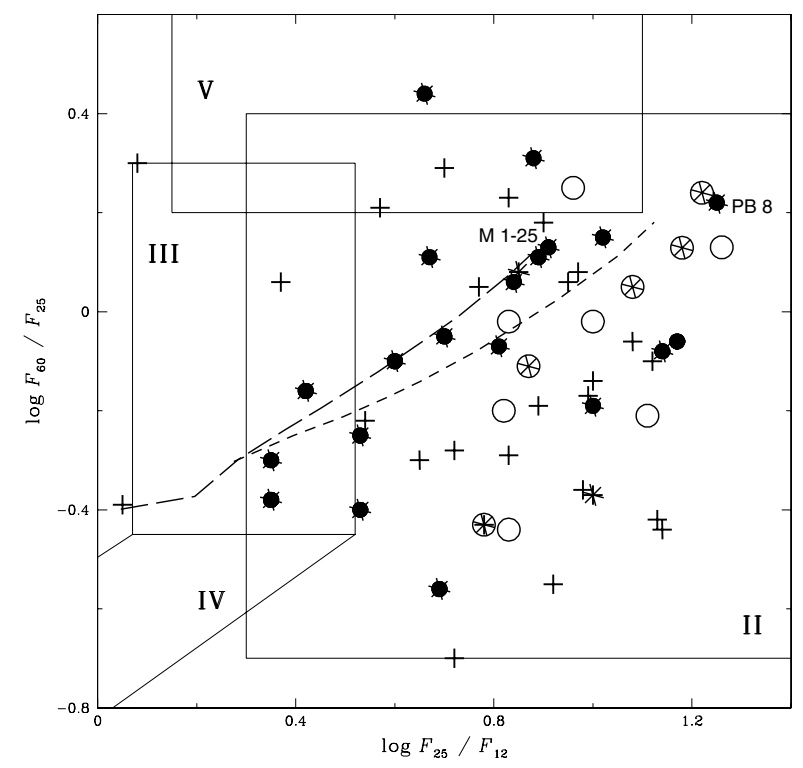

Fig. 7. IRAS colours for the [WR] stars (filled circles, wels (open circles) and non-emission-line stars (plus symbols), with asterisks are overplotted the turbulent PNe. The boxes indicate source classifications for different colours, taken from Zijlstra et al. (2001). Box II are planetary nebulae, box III post-AGB stars, box IV OH-Miras and Box V bipolar outflow sources. The dashed lines show simple evolutionary sequences, explained in the text. Labelled are two individual PNe discussed in Sect. 6.2.

from cool stars with bluer IRAS colours to hot stars with (more evolved) redder IRAS colours is visible for the [WR] stars, but not for the non-emission-line stars and the (less populated) wels group (Zijlstra 2001).

Detection statistics show the differences between the groups. Out of the 101 stars, 60 have three good IRAS detections. The detection rates are 28 out of 57 non-emission-line stars, 12 out of 21 wels, and 20 out of 23 [WR] stars: $49 \%, 57 \%$, and $87 \%$, respectively. This shows that the [WR] stars are on average much stronger IRAS emitters. If we only consider 25 and 60 microns, the detection rates become $89 \%, 86 \%$, and $100 \%$, i.e. the difference is rather reduced. The numbers indicate that [WR] stars are much brighter at 12-micron, but less so at the other bands.

The wels have the same detection rate as other non-[WR] stars. However, all 5 wels with turbulence have three-band detections; their colours are similar to the [WO] stars. All wels without 12-micron detection are non-turbulent objects.

\section{Evolutionary parameters}

\subsection{Temperature distributions}

The temperature distribution of the three groups of stars, defined as in Sect. 4.1, is shown in Fig. 8. The [WR] stars show some clustering at low and high temperatures with a gap in between (see upper panel). This gap has been noted for a long time (see e.g. Gorny 2001). The wels stars, in contrast, peak precisely in this gap. In contrast, the non-emission-line stars show a peak in their distribution at high temperature (see central panel).

Summing the two groups of emission-line stars (central panel), we find a flat distribution (in logarithmic bins!). Summing all three groups together (bottom panel, drawn line) shows an increasing number of objects with $\log T$.

We compare the temperature distribution of the various $\mathrm{PNe}$ samples with what is predicted by the evolutionary tracks of

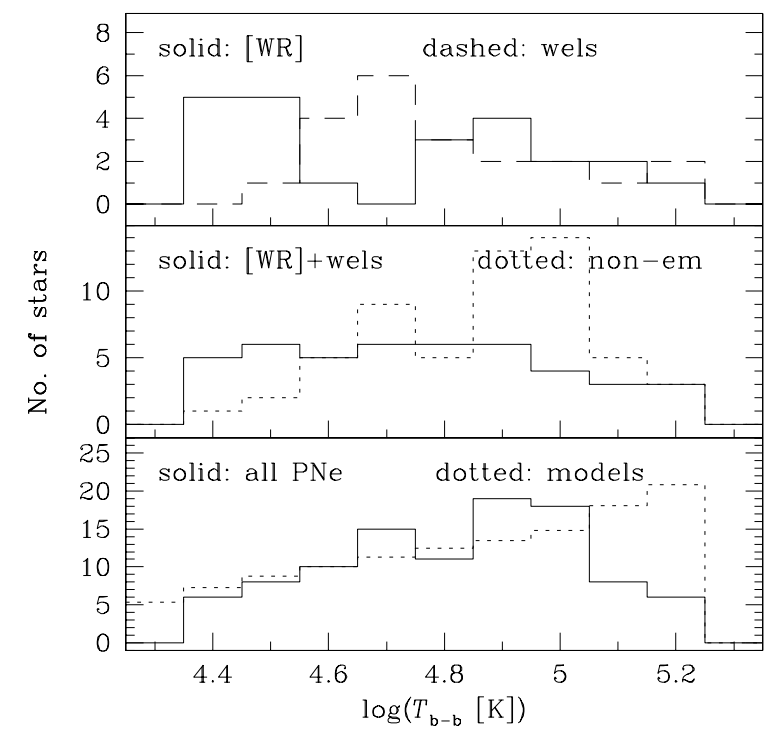

Fig. 8. Temperature distribution of [WR], wels, and non-emission-line stars, in logarithmic temperature bins. The bottom panel, discussed in the text, compares the observed data with the histogram obtained from the H-burning evolutionary models of Blöcker (1995).

Bloöcker (1995). We interpolate the tracks to constant temperature steps, in order to calculate the predicted number of stars per temperature range (proportional to the time spent in the range). The model tracks have to first order a fairly constant temperature increase, $\mathrm{d} T / \mathrm{d} t$. In logarithmic bins, this means that the number of stars per bin increases with temperature. To be consistent with our model analysis we only use the the horizontal part of the evolutionary track, before the knee in the H-R diagram. Figure 8 (bottom panel, dotted line) shows the predicted distribution for hydrogen burning $0.605 M_{\odot}$ track of Blöcker (1995). The predicted number of objects (per logarithmic temperature bin) gradually increases towards higher temperatures, as expected from the increasing width of the bins and from slowing the evolution.

When compared to the observational data, we see that no single group of PNe produces a histogram similar to the models. The solid line in the bottom panel of Fig. 8 shows that the sum of all classes produces a histogram that is the most similar to the theoretically expected distribution. Thus, the full sample is representative for a uniformly sampled Blöcker track. The flat distribution of the emission-line stars implies that this group is biassed towards lower temperatures, relative to standard evolution. As a separate group the wels show a strong temperature preference and cannot represent a full evolutionary track.

It is possible that our observations misrepresent the oldest PNe with the hottest cores. Our sample is mainly restricted to the spherical PNe with an ionization boundary, while for old PNe the ionization front breaks through and may cause fragmentation of a nebula, making it unsuitable for our analysis. We also cannot exclude a systematic error of our $T_{\mathrm{b}-\mathrm{b}}$ values that may shift the maximum of the observed distribution, and this error may also depend on temperature.

\subsection{Dynamical ages of the $P N e$}

Evolutionary tracks should connect objects along sequences of increasing dynamical age and increasing stellar temperature, until the stars reach the knee in the HR diagram, after which the stellar temperature slowly decreases. Helium-burning tracks generally also follow this sequence, but the stars evolve a few 


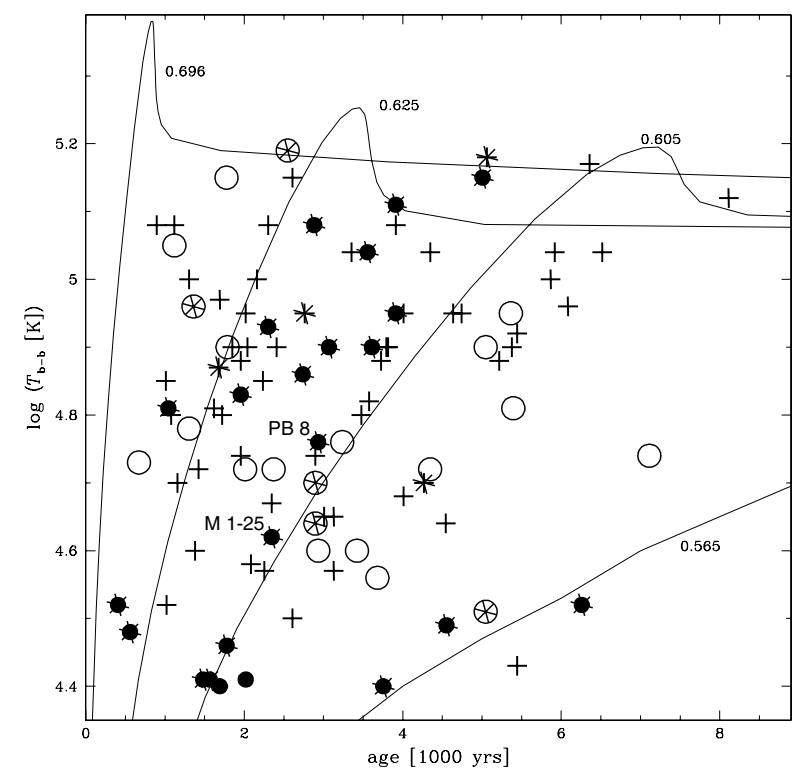

Fig. 9. The age-temperature diagram. Filled circles indicate [WR] stars, open circles are wels, and pluses indicate non-emission-line stars. Turbulent nebulae are indicated by the star symbols. Solid lines show Schönberner's H-burning evolution. Labelled are two individual PNe discussed in Sect. 6.2.

times more slowly (factor of 3 according to Blöcker 1995); the details depend on the precise timing of the thermal pulse preceding the switch to helium burning.

Figure 9 plots the dynamical age versus the temperature for the stars in our sample. The interpolated (hydrogen-burning) evolutionary tracks are overplotted with a solid line. As in Fig. 7, different symbols distinguish types of stars and indicate turbulence.

The figure shows that some regions in this age-temperature plane are dominated by certain types of objects. The [WR] stars are mainly found in the narrow region between the 0.605 and $0.625 M_{\odot}$ tracks, with a few further objects at low temperatures and a range of ages. The wels stars are spread mostly out at intermediate temperatures. The non-emission-line stars tend to avoid the low temperature region, but cover a region in the top-right corner that the other stars tend to avoid.

The statistics of our full sample of $101 \mathrm{PNe}$ shows that the average dynamical age of non-emission-line central stars and of wels is 3200 years, while for [WR]-stars it is 2800 years, with a standard error on the mean of about 300 years in both cases. This difference is not significant, and the result may be contaminated by a selection effect because it is easier to detect a [WR] star at lower temperatures (see Górny et al. 2004).

\subsection{Non-emission-line objects}

In Fig. 9 the non-emission-line objects are mixed with the emission-line ones except for the upper right area. This is the place where the cooling parts of evolutionary tracks of massive stars cross with heating parts of evolutionary tracks of low mass stars. The hot $\left(10^{5} \mathrm{~K}\right)$, non-emission-line stars with old nebulae could be high-mass stars located on the cooling track. The alternative interpretation is that they have lower-mass central stars. In either case, the absence of emission-line stars in this region is obvious. Either a minimum mass is required for a hot [WO] star of about $0.61 M_{\odot}$, or the stellar wind ceases when the star reaches the cooling track. The latter is plausible since the luminosity drops quickly by a factor of 100 at this time. This fact is well known for hot star winds (see e.g. Owocki 1994; Owocki \& Gayley 1995). Koesterke \& Werner (1999) show that PG 1159 stars have much weaker winds than wels stars, and suggest that the wind mass-loss rate decreases rapidly when the star enters the cooling track.

\subsection{Nebular evolution}

Schönberner et al. (2005a) conclude that during evolution the PN material always enters the "champagne phase" where the ionization front breaks through and the PN becomes density bounded. This disagrees with our result that most of the $101 \mathrm{PNe}$ are ionization bounded despite their age and core $T_{\text {eff }}$. In our sample the presence of the ionization boundary was deduced from the strengths of the low-ionization low-excitation lines. A possible explanation of this disagreement can be found in Gesicki et al. (2003), where we compared the model luminosities with those obtained from evolutionary tracks and suggested that "PNe are leaking". This indicates that $\mathrm{PNe}$ are a mixture of regions that are both transparent and opaque for ionizing radiation. The spherical Schönberner's and Torun models cannot investigate asymmetric or clumpy nebulae. However there is another explanation - the ionization boundary is not so very exceptional. The evolutionary sequence No. 4 of Perinotto et al. (2004), with high AGB mass loss rate and low AGB wind velocity, never becomes optically thin and can serve here as a hint. It seems to be promising to follow this idea and to search for other hydrodynamical models that remain ionization bounded for a significant part of PN evolution. In Gesicki \& Zijlstra (2003) and Zijlstra et al. (2005, in preparation) we presented models supported by HST images showing very small central cavities of the nebulae. This finding supports the above idea of a low AGB wind velocity, at least at the end of AGB and for some PNe. Finding an appropriate hydrodynamic model might constrain the still unknown AGB mass-loss history.

\section{The hydrogen-poor sequences}

\subsection{The $[W R]$ stars}

A global nebular quantity, turbulence, is predominant among the [WR] stars. Mellema (2001) links the metal-enriched [WR] winds with a long-lasting momentum-driven phase that drives the turbulence. This confirms that they form a distinct group, and it can be interpreted as evidence of a separate different evolutionary channel. Figures 7 and 9 show that there is a marked uniformity among the majority of the [WR] stars. Figure 9 suggests this is related to the core mass of the star, where stars of a relatively narrow range in core mass account for the majority of the [WR] stars. Caution should be taken when quoting precise masses: the location in the age-temperature diagram depends on modelling assumptions as discussed above.

The [WR] stars are in general hydrogen-poor and are considered helium-burning central stars. These stars will show a slower temperature increase than do hydrogen-burning tracks of the same mass, although the detailed evolution is complicated. The distribution of [WR] stars in Fig. 9 is not entirely inconsistent with such an inclined track, but strong conclusions are not warranted.

There are three [WC]-PNe located near the $0.565 M_{\odot}$ track: from the left M 4-18, He 2-99, and NGC 40. All are old nebulae with cool stars. The first is determined as old because of small expansion velocity, the other two PNe are large $(\approx 0.15 \mathrm{pc})$ as expected for old objects. Their three central stars were analyzed by 
Leuenhagen et al. (1996). All have long been suspected of being born-again PNe, where the central stars have suffered a VLTP, leading to a rejuvenated star inside an old nebula (e.g. Hajduk et al. 2005). Helium-burning stars are not expected to experience another VLTP. The stars along the VLTP sequence therefore represent a separate evolutionary channel from the majority of the [WR] stars. The difference may largely be one of timing, that is, whether the last thermal pulse takes place along the horizontal sequence (LTP) or on the cooling track (VLTP). The only nonemission-line star in this region is the halo object PN G 359.233.5, which could well be a genuine low-mass object (McCarthy et al. 1991). The wels in this region is He 2-108 (its classification is uncertain).

There is a group of 7 [WC] stars that combine very compact nebulae (low ages) with very cool central stars. This group has temperatures in a narrow range around $\log T_{\mathrm{b}-\mathrm{b}}=4.7$, well separated from hotter [WR] objects (see Figs. 5 and 9). The IR-[WC] stars and the mixed-chemistry objects are found here (Zijlstra 2001). It is not clear that these belong to either of the two groups discussed above. The mixed chemistry has been associated with binary evolution (Zijlstra et al. 2001; de Marco et al. 2004), and in one case (CPD-56 8032 - Cohen et al. 1999; de Marco et al. 2002), there are strong indications of binarity. The precise role of a binary companion is not clear, but it may involve retaining old ejecta in a disk, triggering accretion, and possibly providing a source of ionization. They could have similar origins to the R Cor Bor stars (see the review of Clayton 1996), although the very different circumstellar environments suggest the relation is not an evolutionary one.

This group of cool, compact objects could in principle have descendants among the hot [WO] stars, which have more evolved nebulae. However, the mixed chemistry (i.e. evidence of both a neutral oxygen-rich and an inner carbon-rich regions) and unique IRAS colours argues against this. But there are no obvious descendants among the non-emission-line stars either, leaving the evolution of this group unexplained. If the stars are accreting, the extended, cool photosphere could hide a much hotter underlying star, which would explain the lack of intermediatetemperature related stars, but still leaves the question of descendants open.

\subsection{The gap in $[W R]$ temperature distribution}

The lack of PNe cores of [WC 5-7] type is well known (Crowther et al. 1998; Górny 2001). This feature can also be seen in our temperature distributions presented in Figs. 5 and 9. One of the two [WR] star filling this gap is PB 8 (PN G 292.4+04.1) of [WC 5-6] type (Acker \& Neiner 2003). However it was classified by Parthasarathy et al. (1998) as wels, and Mendez (1991) found it to be H-rich. In Fig. 7 this object is positioned among wels stars. Therefore we propose that PB 8 is a wels that no longer fills the [WR] temperature gap. Another object, the only known [WC 6] type - M 1-25 (PN G 004.9+04.9) fits the centre of the gap. The Torun models resulted in a stellar temperature $\log T_{\mathrm{b}-\mathrm{b}}=4.6$ (Gesicki \& Acker 1996). However much higher temperatures have been published for the M 1-25 central star, reaching $\log T_{\mathrm{b}-\mathrm{b}}=4.9$ (Samland et al. 1992) based on the helium ionization. In Fig. 7 this object is placed with other regular [WR] objects. Therefore we propose that M 1-25 is a [WC 6] object (we don't question the WR classification) but its temperature may be above the discussed gap.

The uncertain status of the interlopers strengthens the case that the gap in the temperature distribution of [WR] stars is real and quite prominent: the region $4.55<\log T_{\mathrm{b}-\mathrm{b}}<4.8$ appears devoid of [WR] stars. This supports the proposed separate evolutionary sequences of cool and hot [WR] stars. The same conclusion was reached by Crowther et al. (1998), who applied a unified classification scheme to massive WR and low mass [WR] stars and realized that the gap mentioned above is not visible for WR objects.

\subsection{The wels}

The wels appear to be a mixed zoo. We tentatively identify three groups, beginning with one that shows similarities to the [WR] stars in distribution in Fig. 9. These include one object among the VLTP sequence, and approximately 5 objects that form a lowtemperature extension to the hot [WO] stars and may be their progenitors. Second, about 6 wels are found along the high-mass track $\left(>0.625 M_{\odot}\right)$. We suggest that these are objects where the high stellar luminosity drives a stellar wind; they may be H-rich and not related to the [WR] stars. Finally, there is a group along a low-mass track $\left(M \approx 0.6 M_{\odot}\right)$. These could represent failed [WR] stars, where the luminosity is insufficient for driving a full WR-type wind.

The wels on average show a shift in location in Fig. 6: they fall more in a region that is consistent with a lower power index than do [WR] stars. The nebulae around [WR] stars tend to be more centrally condensed than those of wels stars. This points to a different mass-loss history and is consistent with the suggestion that some fraction of wels, possibly the majority, are not evolutionarily related to the [WR] stars.

The small group that may be [WO] progenitors suggests a sequence wels-[WO], reflecting increasing temperature. This could reflect a bi-stability jump, where the strength of a stellar wind suddenly changes when a change in ionization equilibrium makes different lines act in the absorption of radiation. A similar situation is known among early-type supergiants (e.g. Vink et al. 1999; Tinkler \& Lamers 2002). But Tinkler \& Lamers find that among PNe cores, the wind suddenly decreases by a factor of up to 100 , precisely at the temperature where we find the wels[WO] transition. They attribute this to a CNO bi-stability jump. Their analysis used stars classified as $\mathrm{O}(\mathrm{H})$ only, and could be biassed if hotter stars with high $\dot{M}$ appear as [WO] stars.

For the other stars, the driving factor differentiating wels and [WR] stars appears to be luminosity.

\subsection{The PG 1159 stars}

The hot PG 1159 stars are usually considered the most natural descendants of [WR] stars (Werner 2001). They are not a uniform group: Werner et al. (1996) refer to them as the "PG 1159 zoo". There is also a group of so-called "[WC]-PG 1159 transition objects" that are found between [WCL] and [WCE] groups in the $\log g-\log T_{\text {eff }}$ plane (Werner 2001). Because our results suggest separate sequences for cooler [WC] and hotter [WO], one could envisage that cool [WC] stars evolve towards white dwarfs via a [WC]-PG 1159 phase, whilst hot [WO] stars do so via a PG 1159 phase. However, this remains speculative.

It has been reported that the central star of the Longmore 4 nebula changed from a PG 1159 type to a [WC 2-3], for a period of a few months (Werner et al. 1992). Its absorption spectrum transformed into an emission spectrum, with changes proceeding on a timescale of days. It was interpreted as strongly enhanced mass loss, and may have been triggered by an accretion event. As yet, it is the sole documented example of such impressive variability among $\mathrm{PN}$ cores, but even so it shows that the picture 
is a complicated one, where the chaotic picture of wels could be interpreted in terms of stellar wind activity.

\section{Conclusions}

We have deduced the velocity field for $33 \mathrm{PNe}$. Since this was done in most cases by considering two nebular lines, we only regard the mass averaged value as robust. For six PNe the analysis was based on three or more lines and is therefore more reliable. The discussion of details of the velocity field, e.g. turbulence, should be therefore treated with caution, while the analysis based on the mass averaged velocity (ages, masses) should be reliable. We combined this sample with earlier published data and discussed the full sample of $101 \mathrm{PNe}$.

We found no evidence of an increase in nebular expansion velocity with time. This correlation was checked against $T_{\mathrm{b}-\mathrm{b}}$, which should correspond to stellar age and to the nebular dynamical age i.e. two independent characteristics. The [WR] stars show a tendency to low ratios of $T_{\mathrm{b}-\mathrm{b}} / V_{\text {exp }}$. Comparison with the Schönberner et al. (2005a) models suggests that these objects have more centrally condensed nebulae, indicative of increasing mass-loss rate with time on the AGB. Emission-line stars are not found on the cooling track.

We found a correlation between IRAS 12-micron excess and the [WR] stars. This excess is not shown by the wels. The cause of 12-micron excess is typically a population of small grains. These may form in the wind, or form in the collision between the stellar wind and the nebula. Without exception, the wels do not show any 12-micron excess.

We confirm the strong correlation between turbulence and [WR] stars. However, the correlation is not one-to-one, since there are a few non-emission-line stars with turbulence and a few [WR] stars without. The wels appear to have an intermediate amount of turbulence.

The distribution in the age-temperature plane suggests that there are several groups of [WR] stars and wels. One group shows evolved nebulae with cool stars and probably originates in a VLTP. The hot [WO] stars show a narrow distribution corresponding to a small range in core mass. Some wels fall on the same sequence but at lower temperature, so they may be the [WO] progenitors. Other wels are distributed on higher and on lower mass tracks, which lack [WR] stars. Finally, a group of cool [WC] stars with IR-bright compact nebulae have no counterpart in any other group of PNe.

The relation between the [WR] stars and the wels is therefore a complicated one, with neither group being uniform. For the majority of the wels, there is evidence against their being evolutionarily related to the [WR] stars. There is a noticeable apparent difference in core mass and dust properties and in nebular turbulence. A small group (5 objects) of wels have characteristics that are consistent with [WO] star progenitors. Apart from this wels-[WO] sequence, the wels contain stars at both the high and low luminosity ends of the distribution. The former are probably stars where the strong radiation field drives the wind, but are not necessarily $\mathrm{H}$-poor. The latter may be $\mathrm{H}$-poor, failed [WR] stars where the luminosity is too low to maintain a strong [WR] wind. For the [WO] progenitors, the determining factor appears to be stellar temperature: the particular ionization balance may reduce the efficiency of the radiation-driven wind.

The determined discontinuity in temperatures of [WR]-type stars is in favour of the suggested separate evolutionary channels of cool and hot [WR] objects.
Acknowledgements. This project was financially supported by the "Polish State Committee for Scientific Research" through the grant No. 2.P03D.002.025, by the CNRS through the LEA Astro-PF program, and by a NATO collaborative program grant No. PST.CLG.979726. ESO provided support via its scientific visitor program. The NTT observations were associated with observing program 67.D-0527.

\section{References}

Acker, A., Górny, S. K., \& Cuisinier, F. 1996, A\&A, 305, 944

Acker, A., Gesicki, K., Grosdidier, Y., \& Durand, S. 2002, A\&A, 384, 620

Acker, A., \& Neiner, C. 2003, A\&A, 403, 659

Blöcker, T. 1995, A\&A, 299, 755

Charbonneau, P. 1995, ApJS, 101, 309

Charbonneau, P. 2002, An Introduction to Genetic Algorithms for Numerical Optimization, NCAR Technical Note 450+IA (Boulder: National Center for Atmospheric Research) Clayton, G. C. 1996, PASP, 108, 225

Cohen, M., Barlow, M. J., Sylvester, R. J., et al. 1999, ApJ, 513, 135

Crowther, P. A., De Marco, O., \& Barlow, M. J. 1998, MNRAS, 296, 367

Cuisinier, F., Maciel, W. J., Köppen, J., Acker, A., \& Stenholm, B. 2000, A\&A, 353, 543

de Araujo, F. X., Marcolino, W. L. F., Pereira, C. B., \& Cuisinier, F. 2002, AJ, 124, 464

de Marco, O., Barlow, M. J., \& Cohen, M. 2002, ApJ, 574, 83

de Marco, O., Barlow, M. J., Cohen, M., et al. 2004, in Asymmetrical Planetary Nebulae III: Winds, Structure and the Thunderbird, ed. M. Meixner, J. H. Kastner, B. Balick, \& N. Soker, ASP Conf. Proc. (San Francisco: Astronomical Society of the Pacific), 313, 100 Dopita, M. A., \& Hua, C. T. 1997, ApJS, 108, 515

Dopita, M. A., Meatheringham, S. J., Webster, B. L., \& Ford, H. C. 1988, ApJ, 327, 639 Dreizler, S., Werner, K., Heber, U., \& Engels, D. 1996, A\&A, 309, 820

Frankowski, A. 2003, A\&A, 406, 265

Gesicki, K., \& Acker, A. 1996, Ap\&SS, 238, 101

Gesicki, K., \& Zijlstra, A. A. 2000, A\&A, 358, 1058

Gesicki, K., \& Zijlstra, A. A. 2003, MNRAS, 338, 347

Gesicki, K., Acker, A., \& Szczerba, R. 1996, A\&A, 309, 907

Gesicki, K., Acker, A., \& Zijlstra, A. A. 2003, A\&A, 400, 957

Górny, S. K. 2001, Ap\&SS, 275, 67

Górny, S. K., Stasinska, G., \& Tylenda, R. 1997, A\&A, 318, 256

Górny, S. K., Stasinska, G., Szczerba, R., \& Tylenda, R. 2001, A\&A 377, 1007

Górny, S. K., Stasinska, G., Escudero, A. V., \& Costa, R. D. D. 2004, A\&A, 427, 231

Hajduk, M., Zijlstra, A. A., Herwig, F., et al. 2005, Science, 308, 231

Herwig, F. 2001, Ap\&SS, 275, 15

Koesterke, L. 2001, Ap\&SS, 275, 41

Koesterke, L., \& Hamann, W.-R. 1997, A\&A, 320, 91

Koesterke, L., \& Werner, K. 1999, ApJ, 500, L55

Koza, J. 1992, Genetic Programming (Cambridge: MIT Press 1992)

Leuenhagen, U., \& Hamann, W.-R. 1998, A\&A, 330, 265

Leuenhagen, U., Hamann, W.-R., \& Jeffery, C. S. 1996, A\&A, 312, 167

Marcolino, W. L. F., \& de Araujo, F. X. 2003, AJ, 126, 887

McCarthy, J. K., Mould, J. R., Mendez, R. H., et al. 1990, ApJ, 351, 230

McCarthy, J. K., Rich, R. M., Becker, S. R., et al. 1991, ApJ, 371, 380

Mellema, G. 2001, Ap\&SS, 275, 147

Mellema, G., \& Lundqvist, P. 2002, A\&A, 394, 901

Mendez, R. H. 1991, in Evolution of Stars: The Photospheric Abundance Connection, ed. G. Michaud, \& A. Tutukov, IAU Symp., 145, 375

Owocki, S. P. 1994, in Pulsation; rotation; and mass loss in early-type stars, ed. L. A Balona, H. F. Henrichs, J. M. Contel, IAU Symp., 162 (Dordrecht: Kluwer Academic Publishers), 475

Owocki, S. P., \& Gayley, K. G. 1995, in Wolf-Rayet stars: binaries; colliding winds; evolution, ed. K. A. van der Hucht, \& P. M. Williams, IAU Symp., 163 (Dordrecht: Kluwer Academic Publishers), 138

Parthasarathy, M., Acker, A., \& Stenholm, B. 1998, A\&A, 329, L9

Peña, M., Medina, S., \& Stasinska, G. 2001, A\&A, 367, 983

Peña, M., Medina, S., \& Stasinska, G. 2003a, Rev. Mex. Astron. Astrofis., 15, 38

Peña, M., Medina, S., \& Stasinska, G. 2003b, Rev. Mex. Astron. Astrofis., 18, 84

Perinotto, M., Schönberner, D., Steffen, M., \& Calonaci, C. 2004, A\&A, 414, 993

Rauch, T., Köppen, J., \& Werner, K. 1994, A\&A, 286, 543

Rauch, T., Köppen, J., Napiwotzki, R., \& Werner, K. 1999, A\&A, 347, 169

Sahai, R. 2000, ApJ, 537, L43

Sahai, R., Nyman, L.-̊̊., Wootten, A. 2000, ApJ, 543, 880

Samland, M., Köppen, J., Acker, A., Stenholm, B. 1992, A\&A, 264, 184

Schönberner, D., Jacob, R., Steffen, M., et al. 2005a, A\&A, 431, 963

Schönberner, D., Jacob, R., \& Steffen, M. 2005b, A\&A, 441, 573

Siebenmorgen, R., Zijlstra, A. A., \& Krügel, E. 1994, MNRAS, 271, 449

Tinkler, C. M., \& Lamers, H. J. G. L. M. 2002, A\&A, 384, 987

Tylenda, R., Acker, A., \& Stenholm, B. 1993, A\&AS, 102, 595

Vink, J. S., de Koter, A., \& Lamers, H. J. G. L. M. 1999, A\&A, 350, 181

Weinberger, R. 1989, A\&AS, 78, 301

Werner, K., Hamann, W.-R., Heber, U., et al. 1992, A\&A, 259, L69

Werner, K., Dreizler, S., Heber, U., \& Rauch, T. 1996, in Hydrogen deficient stars, ed. C. S. Jeffery, \& U. Heber, ASP Conf. Ser., 96, 267

Werner, K. 2001, Ap\&SS, 275, 27

Zhang, C. Y., \& Kwok, S. 1991, A\&A, 250, 179

Zijlstra, A. A., Pottash, S. R., \& Bignell, C. 1989, A\&ASS, 79, 329

Zijlstra, A. A., van Hoof, P., Chapman, J. M., \& Loup, C. 1994, A\&A, 290, 228

Zijlstra, A. A., Chapman, J. M., te Lintel Hekkert, P., et al. 2001, MNRAS, 322, 280

Zijlstra, A. A. 2001, Ap\&SS, 275, 79 